\title{
Förderung von informellen Kontexten und Awareness in Scrum-Teams
}

\author{
Jan Schwarzer, Susanne Draheim, Kai von Luck \\ Department Informatik, Hochschule für Angewandte Wissenschaften Hamburg
}

\begin{abstract}
Zusammenfassung
Awareness und informelle Kommunikation sind zentrale Bestandteile einer effektiven Zusammenarbeit in Scrum-Teams. Oft fehlt es jedoch an wichtigen Informationen für die tägliche Arbeit, da diese z. B. in den verwendeten Systemen versteckt oder nicht zugänglich sind. Auch werden informelle Kontexte nur unzureichend technisch unterstützt. Das Ambient Surface-System als ein Ambient Display ermöglicht es, diesen Herausforderungen zu begegnen. Neben der Förderung von Awareness, macht es Informationen in informellen Situationen zugänglich. Dieser Beitrag untersucht eine solche Lösung und ihren Einfluss auf die täglichen Arbeitsprozesse in einem agilen Software-Entwicklungsumfeld. Es wurden quantitative und qualitative Nutzungsdaten erhoben und inhaltsanalytisch sowie statistisch ausgewertet. Präsentiert werden ausgewählte Ergebnisse aus einer seit Februar 2014 andauernden Fallstudie, welche in Kooperation mit einem Projektpartner aktuell durchgeführt wird.
\end{abstract}

\section{$1 \quad$ Einleitung}

Im Forschungsfeld der Computer Supported Cooperative Work (CSCW) hat die Untersuchung von Awareness in ihren unterschiedlichen Ausprägungen mittlerweile eine breite Erkenntnisbasis erbracht (Dourish \& Bellotti 1992; Röcker et al. 2004). Die grundsätzliche Förderung von Awareness erlaubt zum einen die Vermeidung doppelter Arbeitsschritte und zum anderen fein abgestimmte Prozesse (Dourish \& Bellotti 1992). Awareness ist damit ein wichtiger Bestandteil kollaborativer Arbeiten (Röcker et al. 2004). Auch in SoftwareEntwicklungsteams spielt Awareness eine wichtige Rolle (Kraut \& Streeter 1995). Neben der Verständigung auf ein gemeinsames Projektziel sind beispielsweise der regelmäßige Informationsaustausch oder das Abhalten von Meetings typische Arbeitsschritte. Ein großer Unterschied zu Gruppenkontexten, wo gemeinsames Arbeiten und dessen Schritte für jede Person einsehbar sind, ist, dass in der Software-Entwicklung dies durch den Einsatz digitaler Tools nachzubilden versucht wird. Gleichzeitig erhöht dies jedoch deutlich den Aufwand, sich über die stattfindenden Veränderungen in dieser geteilten und hybriden Welt bewusst zu bleiben (Biehl et al. 2007). 
Agile Methoden finden in der Software-Entwicklung zunehmend Verbreitung (Cho 2009). Diese Ansätze versuchen unter anderem durch kurze und iterative Entwicklungszyklen, die Team-Awareness und -Interaktion zu fördern (Biel et al. 2007). Ganz allgemein rücken agile Vorgehensmodelle vermehrt Individuen und ihre Interaktionen in den Fokus und weniger Prozesse und Tools an sich (Cho 2009). Scrum, als ein beliebter Vertreter dieser Methoden, bringt eine Vielzahl von Methoden und Werkzeugen mit sich (Cho 2009). Neben unterschiedlichen Rollen und Verantwortlichkeiten der Team-Mitglieder (z. B. Scrum Master oder Product Owner) sieht Scrum zudem unterschiedliche Modelle zur regelmäßigen (z. B. Sprint Planning) sowie teilweise täglichen (z. B. Daily Scrum) Koordination vor. Gleichwohl fehlt es häufig an Möglichkeiten, die fortlaufenden Aktivitäten von Team-Mitgliedern effektiv sichtbar zu machen (Biehl et al. 2007).

Nach Kraut und Streeter (1995) erlaubt der Einsatz formaler Arbeitstechniken (z. B. Sprachspezifikationen) heutzutage das Entwickeln qualitativ hochwertiger Software-Lösungen. Dennoch helfen diese Techniken nur bedingt, wenn es um die Koordination von Prozessen geht. Es fehlt an Lösungen, die die Unterstützung informeller Kommunikation in den Blick nehmen. Informelle Kommunikation ist der primäre Kanal des Informationsflusses in Software-Entwicklungsabteilungen und erleichtert darüber hinaus den Zugang zu Informationen. In einer Studie zeigen LaToza et al. (2006), dass Software-Entwickler/innen bis zu $40 \%$ ihrer täglichen Arbeitszeit damit verbringen, über produzierten Quelltext in (nicht) geplanten Meetings oder beispielsweise über E-Mail miteinander zu kommunizieren.

Das Ambient Surface-System, eine Kombination aus einer Software-Lösung und einem interaktiven Bildschirm, nimmt sich dieser Herausforderung an und macht gezielt Informationen im sozialen Kontext von Team-Strukturen zugänglich. Dies erreicht es durch (a) die Installation des Systems als ein Ambient Display (Röcker et al. 2004) in das periphere Umfeld der Teams und (b) die Präsentation von relevanten und prozessbezogenen Informationen, die zuvor in Interviews sowie Beobachtungen identifiziert wurden. Seit 2013 entwickelt sich das System in mehreren Teilstudien (z. B. Schwarzer et al. 2013) weiter und ist aktuell seit Februar 2014 im Einsatz bei einem weiteren Projektpartner. Bisherige Untersuchungen zeigen das Potential der Anwendung und den Mehrwert für die tägliche Arbeit der dortigen ScrumTeams. Insbesondere über den in der Literatur (Koch \& Ott 2011) beschriebenen Neuheitseffekt hinaus, zeigt das System einen Nutzen für die Mitarbeiter/innen. Dieser Beitrag fasst ausgewählte Ergebnisse der laufenden Nutzungsstudien des vergangenen Jahres zusammen.

\section{Forschungskontext}

Eine Vielzahl von Forschungsarbeiten im Bereich von CSCW widmet sich bereits der Untersuchung von Ambient-Lösungen in unterschiedlichen Anwendungskontexten. Im Folgenden werden einige ausgewählte Arbeiten vorgestellt und die Schwerpunkte der eigenen Herangehensweise erläutert und abgegrenzt. 


\subsection{Verwandte Arbeiten}

Ein System zur Förderung von Awareness sowie zur Steigerung der Sichtbarkeit und damit Wertschätzung der Arbeit sind so genannte IdeaMirrors (Koch \& Ott 2011). Bei dieser Lösung werden große und interaktive Bildschirme dazu eingesetzt, Innovationsprozesse in Unternehmen zu optimieren. Dies geschieht zum einen durch die aktive Einbindung aller Beteiligten der Wertschöpfungskette in den Prozess der Ideengenerierung und zum anderen durch die Platzierung des Systems im semi-öffentlichen Raum des Unternehmens. Über ein Web-basiertes Formular können Personen eigene Ideen einbringen, welche im Anschluss auf den Bildschirmen sichtbar sind. Über die interaktive Oberfläche können alle Ideenvorschläge eingesehen und bewertet werden. Die grundlegende Motivation ist die Tatsache, dass Innovationen in Unternehmen insbesondere von der Motivation der eigenen Belegschaft, Kunden und Partner abhängen, um letztendlich Produkte wie auch Prozesse zu verbessern.

Die CommunityWall (Snowdon \& Grasso 2002) ist ein System zur generellen Förderung des Informationsaustauschs in Abteilungen. Auch dieses System nutzt eine Web-basierte Plattform zum Beitragen neuer Informationen. Als Basis dient hier ein Vorschlagssystem, welches automatisch um Kommentare und Feedback angereicherte Informationen sichtbar macht. Zwei interaktive Bildschirme an unterschiedlichen Standorten wurden genutzt, um den Zugang zu diesen Informationen zu ermöglichen. Die CommunityWall fokussiert insbesondere das Aufheben von Informationsbarrieren, die durch die Zerstreuung wichtiger Informationen auf unterschiedlichen Systemen in Unternehmen auftreten können.

FASTDash (Biehl et al. 2007) ist beispielsweise eine Anwendung, welche die Sichtbarkeit der Aktivitäten einzelner Team-Mitglieder in der agilen Software-Entwicklung verbessern soll. Grundlage ist hierbei die Randbedingung, dass Software-Entwickler/innen einen Großteil ihrer Arbeitszeit damit verbringen, sich über die Aktivitäten von Kolleg/innen zu informieren. Darüber hinaus fördert dieses System die Möglichkeiten des informellen Austauschs. Erreicht wird dies durch ein in die Entwicklungsumgebung eingebettetes Plug-in, die Nutzung von so genannter Revision Control Software und das Projizieren der erfassten Informationen über einen Beamer im Entwicklungsbüro.

\subsection{Abgrenzung}

In hohem Maße existieren bisher Evaluationsstudien, welche nur in begrenzten Testzeiträumen (z. B. wenige Wochen) Untersuchungen durchführen, so dass die Ergebnisse auf einen längeren Einsatz schwer übertragbar sind (Satyanarayan et al. 2013; McCarthy et al. 2008). Es fehlt an längerfristigen Erfahrungen mit solchen Ambient-Systemen, welche sich im Unternehmenseinsatz nachhaltig durchsetzen können (Koch \& Ott 2011) aber auch an DesignEmpfehlungen für solche Anwendungen (Huang et al. 2006). Uns sind bislang keine Arbeiten bekannt, welche Ambient Displays in Scrum-Teams einsetzen und die sich daraus ergebenen Veränderungen von Arbeitsprozessen sowie die Entstehung neuer Nutzungskontexte in einer Langzeitstudie untersuchen und dokumentieren. Unsere Arbeiten fokussieren sich auf den langfristigen Einsatz von Ambient-Ansätzen im Kontext von Scrum-Teams. Die zugrunde liegende Prämisse der begleitenden Nutzungsstudien ist dabei der anhaltende For- 
schungsbedarf im Bereich von Evaluationsmethoden bei der Nutzung von SoftwareSystemen sowie auch innerhalb der Software-Entwicklung selbst. In jüngerer Zeit gibt es vermehrt Studien, die die Bedeutung von deskriptiven und empirischen Methoden aus der qualitativen Sozialforschung wie etwa (teilnehmender) Beobachtungen sowie Interviews betonen (Tichy \& Padberg 2007). Allerdings sind solche Vorgehensweisen sowohl bei der Datenerhebung als auch in ihrer Auswertung notwendigerweise aufwändig und gehen mit hohem Ressourcenaufwand einher. Gleichwohl ist der Mehrwert von sogenannten mixed methods - und Feldarbeit im Besonderen - nicht hoch genug einzuschätzen. Ergebnisse quantitativer Erhebungen können durch qualitative Verfahren kontextualisiert, mit Nutzer/innen (kommunikativ) validiert werden und bieten damit hohes Erkenntnispotential für die nachhaltige Implementierung von Software-Systemen (Tichy \& Padberg 2007).

\subsection{Ambient Surface-System}

Grundlage für die Untersuchungen ist das Ambient Surface-System. Es besteht aus einer Hardware- (Touch-fähiger Bildschirm) und Software-Komponente, welche für das Selektieren und Visualisieren von Daten zuständig ist. In mehreren Experten-Meetings wurde bis Februar 2014 am System und seinem Informationsangebot gearbeitet. Zwei Ziele haben dabei insbesondere die Abstimmungen und Arbeiten beeinflusst: (1) Die Steigerung der generellen Sichtbarkeit der für die Entwicklungsabteilung wichtigen Informationen und (2) der Zugang zu den Informationen in informellen Kontexten.

Die für die tägliche Arbeit der Scrum-Teams wichtigen Tools (Atlassian JIRA, Atlassian Confluence und Jenkins) wurden untersucht und relevante von nicht relevanten Informationen in Abstimmung abgrenzt. Im Ergebnis stellt das Ambient Surface-System vier unterschiedliche Informationsartefakte zur Verfügung: (1) Aktivitäten (Arbeiten an Vorgängen) aus JIRA, (2) Build-Informationen aus Jenkins, (3) Sprint Chart-Diagramme der Teams aus JIRA und (4) eine Wiki-Seite mit weiterführenden Informationen zu den Vorgängen in JIRA aus Confluence. Alle Inhalte werden automatisch bezogen und in regelmäßigen Abständen aktualisiert. Es bedarf keiner Administration oder aktiven Inhaltsgenerierung, damit das System arbeiten kann. Weiter wird sich verschiedener Mechanismen bedient, um beispielsweise neue Inhalte hervorzuheben (alternative Farbgebungen oder blinkende Effekte). AuBerdem existieren unterschiedlich granulare Informationsebenen, um das Informationsangebot entsprechend aufzubereiten. Die Default-View zeigt Informationen in abstrakter Form, hauptsächlich mit Hilfe von Icons oder farblichen Hervorhebungen. Die Detail-View, welche durch einen Klick auf ein Element der Default-View sichtbar wird, präsentiert hingegen weiterführende Informationen (z. B. Fehlerinformationen aus Jenkins).

Neben einer Ansicht zweier Informationsbereiche (rechts), zeigt Abbildung 1 die derzeitige Installation des Systems (links). Der Projektpartner beschäftigt rund 400 Mitarbeiter/innen und hat sich auf die Produktentwicklung für die Pharma- und Biotech-Industrie spezialisiert. Seit einigen Jahren wird für die fünf Produktteams Scrum als agiles Vorgehensmodell eingesetzt. Trotz der sich daraus ergebenen Vorteile für die Abteilung, existieren weiterhin Herausforderungen. Neben der teilweise geringen Sichtbarkeit von bestimmten Informationen sowie Prozessabläufen, steigt der Kommunikationsbedarf und es bleibt eine permanente Aufgabe, die verschiedenen Scrum-Teams täglich untereinander zu synchronisieren. 
Das Gebäude besitzt ein Unter- und Obergeschoss und bietet Platz für bis zu 70 Mitarbeiter/innen. Personen unterschiedlicher Verantwortlichkeiten arbeiten in diesem Haus. Dazu zählen Software-Entwickler/innen, Scrum Master, Product Owner, die Abteilungsleitung sowie Administrator/innen. Im Untergeschoss (Aula-Bereich) wurde das System in Form eines 46 Zoll großen und Touch-fähigen Monitors in einer Ständerinstallation platziert.
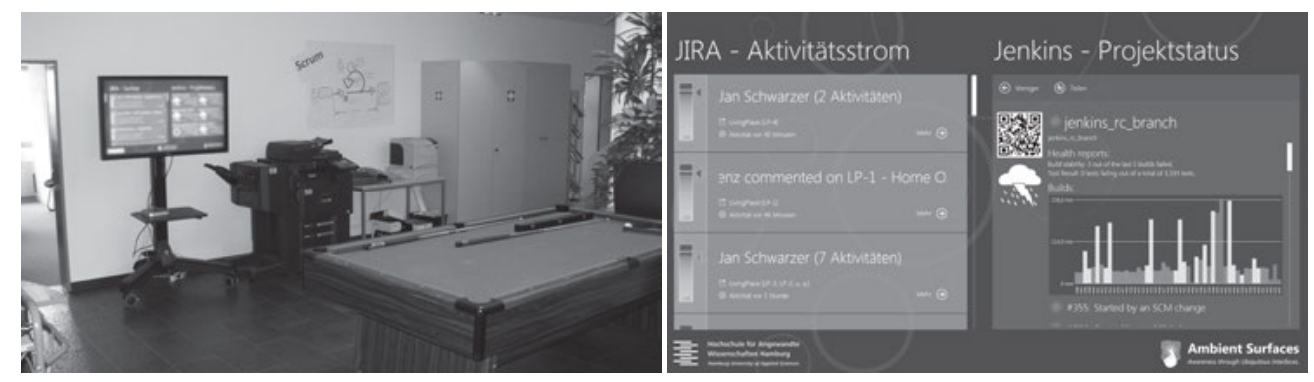

Abbildung 1: Das Ambient Surface-System bei dem Projektpartner im Einsatz (links) und zwei ausgewählte Informationsbereiche (rechts).

Dieser Ort zeichnet sich insbesondere dadurch aus, dass in diesem (a) die meisten Durchquerungen am Tag passieren, (b) sich Getränke, ein Billard-Tisch und Drucker befinden und (c) tägliche Meetings durchgeführt werden. Weiterhin ist dieser Raum der einzige Zugang zu den meisten Büros (gesamtes Obergeschoss), zur Küche und zum Konferenzraum. Durch die Wahl dieser Lokation ist das Ambient Surface-System im sozialen Umfeld den Menschen zugänglich, fern ab vom herkömmlichen Arbeitsplatzrechner.

\section{Forschungsdesign}

Die vorliegende Herangehensweise verbindet methodisch die Erhebung und Auswertung von deskriptiven und quantitativen Nutzungsdaten (d.h. Interaktionsdaten der Anwendung). Auch qualitative Daten, wie Feldnotizen aus teilnehmender Beobachtung, ein inhaltsanalytisch ausgewertetes Experteninterview und Ergebnisse regelmäßiger Gespräche mit Mitarbeiter/innen sowie E-Mails, zählen dazu. Dies macht informelle Kommunikationsprozesse für agile Software-Entwicklung explizit und für die nachhaltige Systementwicklung nutzbar. Die zugrunde liegenden leitenden Fragestellungen der begleitenden Evaluation sind:

1. Wie wird das Ambient Surface-System in der täglichen Arbeit eingesetzt (z. B. Einsatz am Rande von Meetings)? Zentral ist hierbei, wie diese Nutzung in (bestehenden) informellen und spontanen Situationen konkret aussieht.

2. Welche neuen informellen Kontexte können durch den Systemeinsatz ausgemacht werden (z. B. individuelle Informationsaufnahme im Vorbeigehen)?

3. Welchen Einfluss nimmt das System auf die Informationssichtbarkeit? Gibt es z. B. veränderte Arbeitsprozesse aufgrund von sichtbar gemachten Informationen? 
Die bisherigen Datenerhebungen und -auswertungen sind in zwei Phasen durchgeführt worden, die in der Gesamtauswertung in Anlehnung an die Grounded Theory nach Glaser \& Strauss (2010) integriert werden sollen. Dies ist Teil zukünftiger Forschungsaktivitäten. Die in der vorliegenden Fallstudie verwendeten Datenerhebungs- und Auswertungsschritte sind:

- Phase I - Datenerhebung: Erheben von Interaktionsdaten des Ambient SurfaceSystems über insgesamt rund zehn Monate.

- Phase I-Datenauswertung: Begleitende statistische Auswertung und visuelle Aufbereitung von Nutzungsverläufen. Diese bilden die Grundlage für die Erstellung des Leitfadens für das Experteninterview. Das Endergebnis dieser Erhebungsphase ist die inhaltsanalytische Auswertung des Experteninterviews (Mayring 1993).

- Phase II - Datenerhebung: Durchführung teilnehmender Beobachtung (eine Arbeitswoche zu den täglichen Kernarbeitszeiten im Unternehmen). In dieser Beobachtungsphase werden anlassbezogen Ad-hoc-Interviews mit Systemnutzer/innen im direkten Arbeitsumfeld und am Ambient Surface-System selbst durchgeführt.

- Phase II - Datenauswertung: Das Ergebnis dieser Erhebungsphase sind umfangreiche Feldnotizen, aufbereitet in einem Beobachtungsprotokoll (Flick 2012).

\section{Ergebnisse}

Das Ambient Surface-System befindet sich seit Februar 2014 im Einsatz. Dieser Abschnitt präsentiert erste Ergebnisse aus dieser Zeit. Besonderes Augenmerk wird dabei auf Erkenntnisse aus der Phase nach dem einleitend beschriebenen Neuheitseffekt gelegt.

\subsection{Log-Ereignisse}

Zunächst wird ein Einblick in die Nutzung des Systems auf Basis von Interaktionsdaten präsentiert, welche gleichzeitig nur einen Ausschnitt (Veränderungen der aktuellen Sicht) der tatsächlichen Nutzung vor Ort zeigen. Die Auswertung basiert auf der Protokollierung verschiedener Interaktionstypen in einer Log-Datei im Dateisystem. Neben Touchdown-Events (d. h. eine Person berührt die Oberfläche), werden hier Touchmove-Events (d. h. eine Person bewegt ihren Finger über die Oberfläche) sowie Touchup-Events (d. h. eine Person löst den Kontakt mit der Oberfläche wieder) erfasst und unterschieden.

Abbildung 2 zeigt die Nutzung des Systems zwischen den Kalenderwochen 25 und 48 im Jahr 2014. Aufgrund von Krankheitswellen, Urlauben, Schulungen oder Ereignissen, wie Sprint-Planungen oder -abschlüssen, variierte die Nutzung in dem genannten Zeitraum zum Teil sehr stark (vgl. Kalenderwochen 26 und 38). In der gesamten Zeitspanne fielen durchschnittlich 203 Interaktionen (Touchdown-Events) mit dem System pro Woche an. Zum Vergleich: In der Zeit nach der Installation des Systems (KW 8-13) belief sich der Durchschnitt noch auf knapp 2.180 Interaktionen (Touchdown-Events) pro Woche. Grund dafür war vor allem der anfängliche „Entdecker- und Spieltrieb“ (Koch \& Ott 2011). 


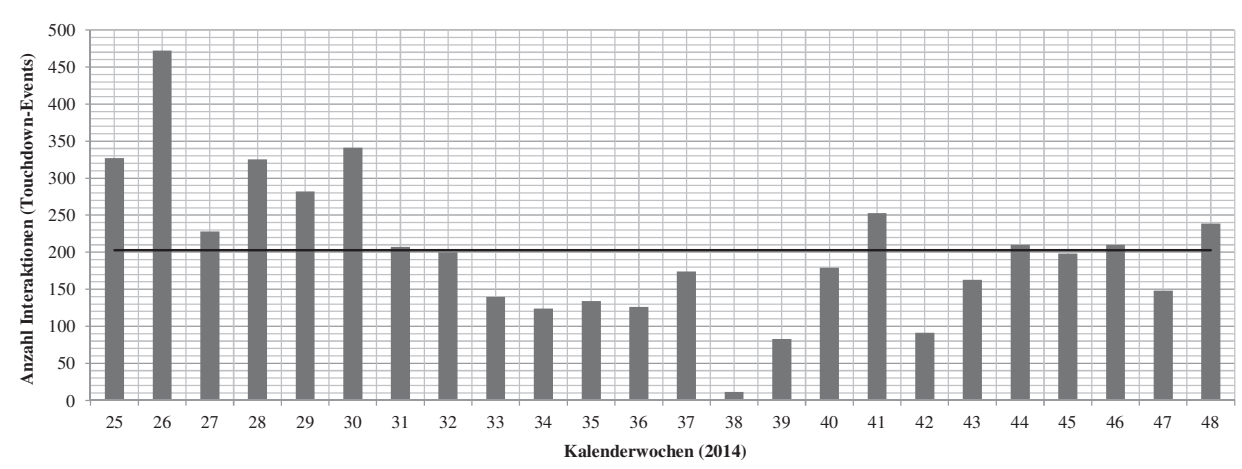

Abbildung 2: Interaktionen (Touchdown-Events) pro Kalenderwoche.

Am häufigsten wurde das System zur Mittagszeit, zwischen 12:00 Uhr und 14:00 Uhr, verwendet. Neben diesem Nutzungshoch, gab es höhere Ausschläge am Morgen (10:30 Uhr) und am Nachmittag (16:00 Uhr), was insbesondere durch Meetings auf dem Flur und spontane Anlässe (z. B. ein Getränk holen) zu begründen ist. In der zuvor genannten Zeitspanne konnten 138.469 Touch-Events (davon 4.865 Touchdown-Events) mit dem System ermittelt werden. Der Tag mit der höchsten Nutzung war der Mittwoch (1.295), gefolgt von Donnerstag (965), Dienstag (960), Freitag (843) sowie Montag (802).

Die insgesamt vier Informationsbereiche des Systems zeigten unterschiedliche Nutzungsmuster auf. So entfielen $42 \%$ aller Interaktionen auf das Hauptfenster. Dies ist dadurch zu begründen, dass die derzeit gewählte Darstellung der Informationen mehr Platz einnimmt, als der Bildschirm in der Breite (1920 Pixel) zur Verfügung stellt. D. h. in manchen Fällen war ein manuelles Scrollen durch das Informationsangebot in horizontaler Richtung notwendig. Die restlichen $58 \%$ verteilten sich wie folgt: $19 \%$ JIRA-Aktivitätsströme, $7 \%$ Jenkins Build-Ansicht, $15 \%$ Sprint Chart-Diagramme und $17 \%$ Confluence-Seite. In den meisten Fällen war die Default-Ansicht eines Informationsbereichs ausreichend. Lediglich die Jenkins Build-Ansicht zeigte im Vergleich eine höhere Nutzung der Detail-Ansichten, in welchen insbesondere für die Entwickler/innen wichtige und detailliertere Informationen (z. B. Changesets) zu den Builds sichtbar wurden.

Das Ambient Surface-System wurde 2014 in mindestens 1.824 unterschiedlichen Situationen eingesetzt $(\approx 10,8$ pro Tag). Grundlage hierbei war die Unterteilung des Zeitstrahls der an 169 Tagen ermittelten Interaktionen (22.683 Touchdown-Events) in 5-Minutenblöcke. Ein einzelner Block enthielt dabei immer mindestens eine Interaktion mit dem System. In $20 \%$ der Fälle folgte ein weiterer Interaktionsblock binnen der nächsten fünf Minuten. Die Ergebnisse dieser Datenreihe liegen im Mittel um ca. 46 Minuten auseinander. Der Median beträgt in etwa 24 Minuten, die Standardabweichung beläuft sich auf ungefähr 58 Minuten. Diese Ergebnisse sind dadurch zu erklären, dass an manchen Tagen Interaktionen um mehrere Stunden auseinanderlagen - an anderen wiederum dicht zusammenhingen. Häufungen sind beispielsweise zur Mittagszeit aber auch am Morgen oder Nachmittag feststellbar. Längere Zeiträume der Inaktivität (z. B. Nächte oder Wochenenden) wurden bei dieser Berechnung ausgenommen, um unnötige Verzerrungen auszuschließen. 


\subsection{Feldforschung}

Da die Log-Ereignisse für sich genommen nur einen Auszug der konkreten Nutzung zeigen (aktive Interaktionen), wurde im Frühjahr 2015 eine erste Beobachtungsphase über fünf Werktage inklusive Gesprächen mit Mitarbeiter/innen sowie ein Gruppeninterview durchgeführt. Ziel war es, neue Nutzungskontexte und Entwicklungspotentiale sichtbar zu machen, die aus den bislang vorwiegend quantitativen Erhebungen nicht hervorgingen.

Der gewählte Installationsstandort des Systems erwies sich als günstig. An zwei Werktagen konnten in knapp 14 Stunden über 1.100 Durchquerungen des Bereichs unmittelbar vor dem System protokolliert werden. Es wurde weiterhin ersichtlich, dass in dem Beobachtungszeitraum das System mindestens so oft passiv wie aktiv eingesetzt wurde. D. h. die tatsächliche Anzahl der Situationen, in welchen das System eingesetzt wurde, übersteigt die Zahl, die durch die ausschließliche Betrachtung der Interaktionsstatistiken feststellbar ist. Die Nutzungsdauer in solchen passiven Szenarien belief sich dabei auf wenige Sekunden bis hin zu einigen Minuten. Ob kurze Blicke beim Vorbeigehen aber auch Situationen, in welchen Personen vor dem Bildschirm stehenblieben, konnten mehrfach beobachtet werden. Auch Zweiergruppen blieben immer mal wieder vor dem Bildschirm stehen (z. B. in der Mittagspause), um über die Inhalte zu sprechen. Es gab außerdem Personen, die das System häufiger nutzten als andere. In Meeting-Situationen wurde zudem hin und wieder das Informationsangebot beiläufig beobachtet oder aktiv auf sichtbare Informationen eingegangen.

Weiterhin sind es vor allem die spontanen Situationen des Arbeitsalltags, in denen das System vorrangig verwendet wurde und weniger geplante oder regelmäßig stattfindende Events. Damit sind beispielsweise das Holen eines Getränks aus der Küche oder das zufällige Zusammentreffen im Flur gemeint. Das System motivierte in der Vergangenheit immer wieder neue Gesprächssituationen. Grund dafür waren beispielsweise humorvoll gemeinte Kommentare zu den Sprint-Fortschritten anderer Teams oder auch gescheiterte Build-Prozesse. Mehrere Personen unterschiedlicher Rollen gaben in Gesprächen an das System grundsätzlich nicht mehr missen zu wollen und betonten den Nutzen für die tägliche Arbeit.

In Ad-hoc-Gesprächen konnte zudem festgestellt werden, dass die Ansprüche an das System rollenabhängig variieren können. Während Personen aus der Software-Entwicklung den Fokus auf Informationen aus dem Jenkins-System legten (z. B. Informationen über fehlgeschlagene Builds), interessierten sich die Abteilungsleitung oder auch die Scrum Master mehr für abstraktere und aggregierte Informationen (z. B. Sprint-Fortschritte).

\subsection{Diskussion}

Der grundsätzliche Mehrwert des Ambient Surface-Systems ist auf mehreren Ebenen feststellbar. Dies spiegeln die Nutzungsstatistiken der Log-Erhebungen aber auch die (teilnehmende) Beobachtung und die geführten Gespräche wider. Das System wird vor allem in spontanen Alltagssituationen verwendet, eben dann, wenn Mitarbeiter/innen gerade nicht an ihrem Arbeitsplatzrechner sitzen. Dies ist oftmals zu den Morgenstunden, zur Mittagszeit und am späten Nachmittag der Fall. Auch sorgt es für neue Gesprächsanlässe, direkt am System oder im Nachhinein durch zuvor wahrgenommene Informationen und aktives Zuge- 
hen auf Personen. Diese Gespräche sind sowohl fachlicher Natur, können aber auch einen Gossip-Charakter besitzen. Das System steigert die Sichtbarkeit von Informationen, begründet durch das Anzeigen von sowohl Team-internen als auch Team-übergreifenden Informationen und deren „Live“-Status. Gleichzeitig ersetzt es aber nicht die täglichen formalen Abstimmungen (z. B. Daily Scrums) in den Teams untereinander. Es zeigt sich, dass das System vor allem die nicht-formalen Arbeitsprozesse im Unternehmen mitgestaltet und den Austausch untereinander fördert, vorrangig gesteuert durch den Zufall der spontanen Nutzung.

Kontinuierliche Veränderungsprozesse in der Software-Entwicklungsabteilung (Einsatz von Tools, Team-Strukturen, Abläufe etc.) geben immer wieder Anlass dazu, das System kontinuierlich den Bedürfnissen und Prozessen anzupassen. So werden in Rücksprache regelmäBig Änderungen an den bestehenden Informationsbereichen durchgeführt, wie auch neue hinzugefügt. Diese begleitenden Arbeiten am System wirken sich grundsätzlich positiv auf die Nutzungshäufigkeit aus. Wichtig ist dabei, gewohnte Aufbereitungen von Informationen aus den täglich genutzten Tools bei deren Darstellung auf dem System entsprechend zu berücksichtigen. Es ist auch entscheidend, Personen beim Vorbeigehen mehr die für sie interessanten Informationen zu präsentieren, macht es aber gleichzeitig schwierig, das zufällige Finden insbesondere Team-übergreifender Informationen zu unterstützen. Ein weiteres Resultat der Untersuchungen ist die geplante Installation eines zusätzlichen Bildschirms, um so a) dem informationsabhängigen Bedarf der verschiedenen Teams besser gerecht zu werden und b) mehr Raum für die Aufbereitung des Informationenangebots zu haben.

\section{Schluss}

Dieser Beitrag diskutiert den anhaltenden Langzeiteinsatz einer Ambient Display-Lösung im Kontext von Scrum-Teams, welcher durch qualitative wie auch quantitative Verfahren der Evaluation begleitet wird. Herausforderungen in Bezug auf die Awareness und informelle Kommunikation machen den Bedarf solcher Lösungen in agilen Software-Entwicklungsabteilungen deutlich. Zukünftige Untersuchungen sollen dabei helfen, das Ambient SurfaceSystem weiter zielführend zu entwickeln und den praktischen Nutzen zu erhöhen. Im Vordergrund steht dabei auch der Einsatz weiterer Erhebungsverfahren, wie beispielsweise EyeTracking, um die Erkenntnisse weiter zu fundieren. Neben einer Intensivierung der aktuellen Zusammenarbeit, existiert das grundsätzliche Bestreben nach neuen Kooperationen mit Scrum-Teams anderer Unternehmen.

\section{Danksagung}

Wir bedanken uns ganz herzlich bei unseren Projektpartner für die konstruktive Zusammenarbeit und hoffen auf eine weiterhin spannende und gleichzeitig anregende Kooperation.

\section{Literaturverzeichnis}

Biehl, J. T., Czerwinski, M., Smith, G. \& Robertson, G. G. (2007). FASTDash: a visual dashboard for fostering awareness in software teams. In Proc. SIGCHI Conference on Human Factors in Computing Systems (CHI '07). ACM, New York, NY, USA, S. 1313-1322. 
Cho, J. J. (2009). An Exploratory Study on Issues and Challenges of Agile Software Development with Scrum. Ph.D. Dissertation. Utah State Univ., Logan, UT, USA. Advisor(s) David H. Olsen.

Dourish, P. \& Bellotti, V. (1992). Awareness and Coordination in Shared Workspaces. In Turner, J. \& Kraut, R. (Hrsg.): Proc. Intl. Conf. on Computer-Supported Cooperative Work. Toronto, Canada: ACM Press, S. 107-114.

Flick, U. (2012). Qualitative Sozialforschung - Eine Einführung. 5. Aufl. Reinbeck: Rowohlt Verlag.

Glaser, B. G. \& Strauss, A. L. (2010). Grounded Theory - Strategien qualitativer Sozialforschung. 3. Aufl. Bern: Hans Huber Verlag.

Huang, E. M., Mynatt, E. D., Russell, D. M. \& Sue, A. E. (2006). Secrets to Success and Fatal Flaws: The Design of Large-Display Groupware. IEEE Comput. Graph. Appl. 26, 1 (Jan 2006), S. 37-45.

Koch, M. \& Ott, F. (2011). CommunityMirrors als Informationsstrahler in Unternehmen. InformatikSpektrum Volume 34, Issue 2, S. 153-164.

Kraut, R. E. \& Streeter, L. A. (1995). Coordination in software development. Commun. ACM, 38(3), S. 69-81.

LaToza, T. D., Venolia, G. \& DeLine, R. (2006). Maintaining mental models: a study of developer work habits. In Proc. of the 28th international conference on Software engineering (ICSE '06). ACM, New York, NY, USA, S. 492-501.

Mayring, P. (1993). Qualitative Inhaltsanalyse - Grundlagen und Techniken. 4. erw. Aufl. Weinheim: Deutscher Studien Verlag.

McCarthy, J. F., Congleton, B. \& Harper, F. N. (2008). The context, content \& community collage: sharing personal digital media in the physical workplace. In Proc. of the 2008 ACM conference on Computer supported cooperative work (CSCW '08). ACM, New York, NY, USA, S. 97-106.

Röcker, C., Prante, T., Streitz, N. \& van Alphen, D. (2004). Using Ambient Displays and Smart Artefacts to Support Community Interaction in Distributed Teams. In Proc. of the OZCHI Conference 2004. Australia: University of Wollongong.

Satyanarayan, A., Strazzulla, D., Klokmose, C., Beaudouin-Lafon, M. \& Mackay, W. (2013). The CHI 2013 Interactive Schedule. In CHI EA '13: ACM Extended Abstracts on Human Factors in Computing Systems. ACM, S. 2987-2990.

Schwarzer, J., Barnkow, L., Kastner, P. \& von Luck, K. (2013). Hin zu mehr Sichtbarkeit und Wertschätzung in der Softwareentwicklung. In Boll, S., Maaß, S. \& Malaka, R. (Hrsg.): Mensch \& Computer 2013: Interaktive Vielfalt. München: Oldenbourg Verlag, S. 341-344.

Snowdon, D. \& Grasso, A. (2002). Diffusing information in organizational settings: learning from experience. In Proc. SIGCHI Conference on Human Factors in Computing Systems (CHI 'O2). ACM, New York, NY, USA, S. 331-338.

Tichy, W. \& Padberg, F. (2007). Empirische Methodik in der Softwaretechnik im Allgemeinen und bei der Software-Visualisierung im Besonderen. In Bleek, W.-G., Raasch, J. \& Züllighoven, H. (Hrsg). Software Engineering 2007 - Beiträge zu den Workshops Fachtagung des GI-Fachbereichs Softwaretechnik (27.-30.03.2007). Hamburg: Gesellschaft für Informatik e.V., S. 211-222.

\section{Kontaktinformationen}

\{ Jan.Schwarzer I Susanne.Draheim \}@ haw-hamburg.de, Luck@informatik.haw-hamburg.de 